\title{
Public health research in times of austerity
}

\author{
GianLuca Quaglio • Ole F. Olesen
}

Published online: 31 July 2011

(C) Springer-Verlag 2011

Public health has been defined as "the science and art of preventing disease, prolonging life and promoting health through organised efforts of society" (Acheson 1988). Most governments have long recognised the importance of public health sciences in reducing the incidence of disease and disability, but public health studies are increasingly used to evaluate the benefits, costs, acceptability, and wider impacts of public health interventions with a view to optimise the cost-effectiveness. Health expenditure in the countries of the European Union has risen faster than the gross domestic product since 1970 (Busse 2001), and cost containment has therefore become the driving force in most health policy discussions (Mossialos and Le Grand 1999). This trend has become even more pronounced following the global financial crisis and the introduction of austerity measures in many industrialised countries.

Public health scientists are therefore challenged to find new ways of gathering data and developing innovative methodologies to quantify the value of public health interventions. Increasing availability of large amounts of data from health surveys, disease surveillance, and large, long-term population-based studies has driven many of the methodological developments in public health (Fielding 1999). Developing new and more complex methodologies to analyse additional aspects of public health will often require input from several scientific disciplines. Rather than a

G. Quaglio · O. F. Olesen $(\bowtie)$

Health Directorate, Directorate General for Research

and Innovation, European Commission,

1049 Brussels, Belgium

e-mail: ole.olesen@ec.europa.eu limitation, this should be seen as an opportunity to evolve the field of public health research by bringing scientists with different backgrounds together. This trend is also apparent throughout this issue of the Journal of Public Health.

The study carried out by Gomes et al. aimed to characterise hand washing among 13-year-old adolescents, assessing the association with the occurrence of diarrhoea. They evaluated 2,036 adolescents enrolled in schools in Porto, Portugal, and found that low frequency of hand washing after toilet visits, before handling food, and before meals was associated with an increased probability of diarrhoea. These results support the hypothesis that improved hand washing habits in adolescence could contribute to a reduction of diseases related to faecal-oral transmission, even in a developed country.

In response to increased case numbers of meningococcal group $\mathrm{C}$ disease, catch-up vaccination strategies have been shown to be successful. Romero et al. describe the results of a repeat vaccination program in Galicia, Spain, where a total of 286,000 subjects were vaccinated, resulting in global vaccination coverage of $82.2 \%$. The incidence of meningococcal disease serogroup $\mathrm{C}$ by season was subsequently reduced from 0.84 cases per 100,000 in 2004/2005 to 0.76 cases per 100,000 in $2005 / 2006$ and 0.18 cases per 100,000 in 2007/2008. In parallel, mortality decreased from eight cases during 2005/2006 to a single case in 2007/2008 . They concluded that the vaccination campaigns against meningococcal group $\mathrm{C}$ disease in Galicia had been successful and resulted in a marked reduction in disease incidence in the vaccination cohort, as well as in the overall population.

The influence of the environment on physical activity has gained popularity as a topic for research during the last 
decade. A variety of tools, including questionnaires, has been developed to measure the perception of the physical environment, but most studies have so far been conducted in the US and Australia. Bucksch and Spittaels tested whether the recently developed ALPHA (Instruments for Assessing Levels of Physical Activity and Fitness) environmental questionnaire is a reliable and valid instrument to assess perceptions of the physical environment in a German population. They concluded that the German version of the ALPHA questionnaire is a useful tool to assess the perceived environment of a neighbourhood for young adults.

Accreditation of medical facilities is currently being tested in Europe and elsewhere as a way to assure and improve the quality of health care. In order to generate ideas for the improvement of German accreditation programmes, Auras and Geraedts explored similarities and differences between nine international approaches to the accreditation of ambulatory primary and specialist care facilities. Accreditation programmes from Germany, Switzerland, the Netherlands, Great Britain, the USA, and Australia were included. The comparison revealed considerable similarities with regard to the development of accreditation criteria, the criteria themselves, and the process of accreditation.

An increasing number of patients use emergency departments, but a vast number of these visits may not be genuinely justified. Rissbacher and colleagues have tried to identify the reasons why people prefer to attend emergency departments. Potential predictors of justified or non-justified visits respectively were identified from 630 respondents to a questionnaire in an average-sized Austrian hospital. Factors such as how a patient was referred to the emergency department, distance, arrival time, formal education, weekday, expected waiting time, previous experiences with the department, emergency, and age were found to influence the number of non-justified visits. The authors applied these findings to develop a useable algorithm that can be used to predict whether or not a visit is justified.

The Framework Programmes for Research and Technological Development (FP) are the European Union's funding programmes to support research projects in Europe. The study presented by Quaglio et al. analysed the features of external experts involved in evaluating the research proposals in FP6 (years 2003-2006) in the area of Life Sciences. The study showed that the experts involved were balanced with respect to nationalities, whereas the gender balance was lower than the pre-determined target. There was a satisfactory degree of rotation of experts between evaluation rounds. However, the percentage of experts from industry was lower than expected. The authors concluded that the evaluation process can and should be studied quantitatively in order to highlight merits, identify weaknesses, and devise improvements to peer-review of research.
There is growing evidence that irregular meal intakes can increase the risk of overweight, enhance insulin resistance, and raise fasting lipid profiles. Muff et al. explored the associations between sociodemographics, health conditions, health behaviours, and work characteristics with irregular meal intakes. This led the authors to hypothesise that indicators of social support, such as number of household members or marital status, other health behaviours, and work conditions, are influencing the regularity of meal intakes to a larger extent than socioeconomic conditions per se. For future public health interventions, they recommend that the issue of irregular meal intakes should be taken into account when promoting healthy nutrition.

With the growing obesity epidemic, there has been a growing concern about the effect of overweight on a number of health outcomes, including the quality of sleep. The research presented by Grandner and colleagues was designed to investigate whether obesity and diabetes diagnosis predict sleep disturbance and daytime fatigue and to what extent, if any, exercise attenuates such a relationship. They concluded that obesity and diabetes are significant independent predictors of sleep disturbance and daytime complaints. Moreover, the relationship with obesity is notably stronger in women than men. A broad measure of exercise (any at all in the past 30 days) was a significant predictor of absence of both sleep disturbance and daytime complaints.

Breast cancer is the most common cancer among Malaysian women, and this spurred Matalqah et al. to compare the health-related quality of life (HRQoL) of breast cancer survivors (BCSs) with that of the general female population in Malaysia. A comparative cross-sectional study using EuroQoL-5 Dimensions (EQ-5D) was conducted at Penang General Hospital in Malaysia. A sample of 150 female BCSs and an equal number from the general population (matched with age bracket and ethnicity) were examined. Findings highlighted that overall HRQoL was lower among BCSs, with more difficulties being experienced across all health dimensions measured by the EQ-5D except mobility. The most significant declines in health status were observed for pain and depression.

Differences in the life expectancy of men and women are a well-known fact. Several theories have been proposed, and a wide range of underlying factors has been examined, but none of these can fully explain the phenomenon. Flandorfer and Fliegenschnee used a qualitative approach to cast further light on this intriguing question. Expert interviews were conducted with professionals in the medical field in Austria about the lifestyles and health behaviour of men and women. The aim was to gain insights as to why the gender gap in mortality has recently been narrowing in many countries. The study revealed that men and women have a 
different understanding of illness and behave in different ways with regard to seeing doctors. However, recent changes in gender roles and changing norms mean that these health behaviour patterns have started to diminish, and this may partly explain the narrowing gender gap in life expectancy.

Taken together, the studies in the present issue provide a glimpse of the width and breadth of public health research. They demonstrate that protecting and promoting the health of populations in a sustainable way require willingness to measure, quantify and evaluate all the variables involved in healthcare and health systems.

\section{References}

Acheson D (1988) Committee of inquiry into the future development of the Public Health Function. Public Health in England, London, HMSO

Busse R (2001) Expenditure on health care in the EU: making projections for the future based on the past. HEPAC Health Econ Prev Care 2:158-161

Fielding JE (1999) Public Health in the 20th century. Annu Rev Public Health 20:xiii-xxx

Mossialos E, Le Grand J (1999) Health care and cost containment in the European Union. Ashgate, Aldershot 